\title{
THE ROLE OF SUBCORTICAL STRUCTURES IN CONDITIONED REFLEXES
}

\author{
Robert W. Doty \\ Department of Physiology, University of Michigan, Ann Arbor, Mich.
}

Anokhin has made two major points of great importance for theories of higher nervous activity. First he has emphasized that in complex, learned behaviors the cerebral cortex is not acting alone, but is in close association with subcortical systems. With the rich interchange known to exist between these systems it is appropriate to seek the mechanism of conditioned reflex formation and performance in the cortical-subcortical relationships rather than predominantly in the cortex itself. While pointing out this importance of subcortical structures, Anokhin has also clearly demonstrated that they are intricately organized and cannot be regarded simply as an indiscriminate "energizing influence" on the cortex. His experiments repeatedly and convincingly documented this second point. Together with many other facts they should convince us that the medial brain stem systems are capable of more precise action than is indicated in their diffuse or "nonspecific" effect upon the EEG.

I am certainly in agreement with Anokhin on both of these points. Thus, rather than discuss details of his paper or simply restate his case, I hope it will be more valuable to offer a summary of data, for brevity and convenience taken chiefly from our laboratory, which give general support to his view and are relevant to his consideration of the role of subcortical structures in conditioned reflexes.*

Using a defensive conditioned reflex (CR) in cats, we found during the course of training that EEG arousal reactions to an auditory conditional stimulus (CS) always attained a high level of consistency before the first respiratory or flexion CR appeared. ${ }^{1}$ Prima facie this indicates participation of subcortical mechanisms, although obviously the effect might be initiated from the cortex. Graphs of the per cent occurrence of these EEG arousal reactions had the form of typical "learning curves" for habituation, conditioning, and extinction. Surprisingly, however, under the conditions of these experiments "habituation" of the arousal reaction to the CS seemed to take place during the early training, even though a shock to the leg was being paired with the CS. Another unexpected finding was that the intensity of the arousal reaction diminished after the CRs became consistent, and with continuation of training a state was reached at which leg flexion CRs could be made with no apparent alteration in the electrical activity of postcruciate, marginal, and middle ectosylvian gyri.

Pursuing this matter independently, Edward Beck and his colleagues at the University of Utah ${ }^{2}$ have found with sleep-deprived cats that when such flexion CRs are made in the absence of low-voltage fast activity in the neocortex, a change in activity is still always found in certain subcortical areas. The

* The original work described in this discussion was performed in the laboratories of the University of Michigan under Research Grant B-1068 from the National Institute of Neurological Diseases and Blindness, Public Health Service, Bethesda, Md., and by a research grant from Foundations' Fund for Research in Psychiatry, New Haven, Conn. 
mesencephalic reticular formation is one of the areas in which such alteration occurs, and is sometimes the first to change its pattern of activity. More often, however, the changes prefacing CR performance occur in the hippocampus and septal areas, and precede those in the mesencephalic reticular formation.

It has been found that defensive CRs can be obtained after destruction of the central core of the mesencephalon, including the reticular formation, or after destruction of the posterior hypothalamus and central thalamus in the stereotaxic planes A-10 to A-12. ${ }^{8}$ In one animal however, there was some indication that certain subcortical structures may be indispensable for this type of conditioning. This animal responded well preoperatively but, following destruction of about $100 \mathrm{~mm}^{3}$ of the medial brain stem, including the mammillary bodies, center median, field $\mathrm{H}_{1}$ of Forel, and the habenulopeduncular tract, the animal made no CRs in 1325 trials in 26 training sessions over 37 days. EEG patterns were within normal limits after the third week, but likewise showed no evidence of conditioned reactions. This animal, although it failed to give CRs, was no more debilitated postoperatively than others with different lesions, which responded adequately. The negative findings thus appear significant.

Since it is difficult to maintain such animals with large bilateral subcortical lesions and since the poor condition of the animal makes it difficult to interpret negative data, Lester Rutledge and I have devised a somewhat different technique for seeking the "indispensable" subcortical structures. In cats trained to flex a leg to a tone, the hippocampal and anterior commissures and the corpus callosum are cut and a large lesion placed subcortically on one side. Direct electrical stimulation of homotopic points on the cerebral cortex is then used as CS, and CR performance is compared for stimulation of the intact side and that with the subcortical lesion. Lesions that seriously encroach upon the internal capsule make stimulation of the ipsilateral middle ectosylvian gyrus ineffective as CS. However lesions confined to the medial geniculate body or transecting and unilaterally destroying the medial thalamus, hypothalamus, and mammillary body in the stereotaxic planes A-7 to A-10 have not altered the effectiveness of the middle ectosylvian gyrus CS.

Although destruction of the habenulopeduncular tract in the latter cases has thus far not been adequate to permit full comparison with the bilateral lesion described above, one is nevertheless forced to consider the possibility that, should there be an "indispensable" brain stem region, it may be activated by diffuse subcortical commissural pathways. Corneliu Giurgea (personal communication) in 1957 on one dog had been able to link a CS electrically applied to one hemisphere to a "US" applied to the motor analyzer of the other hemisphere, even though the entire brain had been completely split from the frontal pole to the anterior border of the mesencephalon. Following section of the corpus callosum, Rutledge and $\mathrm{I}^{9}$ have also found no difficulty in producing CRs in limbs ipsilateral to the cortically applied CS.

When electrical stimulation of the cerebral cortex is used as a CS, undercutting the stimulated zone at first destroys the effectiveness of the CS, whereas circumsecting most of the stimulated cortex does not.,10 Thus a direct projection into subcortical circuits seems to be more important here than intracortical elaboration. 
Once a $C R$ is established to one stimulus modality or location of central stimulation, it is usually readily elicited by other stimuli either immediately or with very little training. ${ }^{9}$ Sarah Southwick and I (in unpublished observations) have confirmed this tendency for stimulus generalization or transfer of training when stimuli are applied almost any place in the brain stem of the cat. Highly similar results have been obtained by Nielson et al. ${ }^{18}$ In rare instances ${ }^{17,18}$ the central stimulation is ineffective, perhaps because of unrecognized technical difficulties. A situation of great interest also arises when the central CS in highly trained animals remains ineffective for a training period of several days or weeks and then rather suddenly begins to elicit CRs. ${ }^{5,9}$ Such cases are uncommon, however, and it seems safe to say that with adequate training electrical stimulation applied any place within the nervous system can elicit a CR. In other words, there are no "silent" areas in this regard. This conclusion can be drawn from extensive data on widely varying placements on the cat cerebral cortex, ${ }^{7,9}$ from about 52 subcortical placements by Nielson et $a l .,^{18}$ and from more than 30 subcortical placements in the unpublished studies of Southwick that ranged from the trapezoid body to the septal nuclei. The same conclusion appears to hold for the monkey (Macacus irus), in which stimulation of such varied regions as the occipital lobe, the precentral, postcentral, marginal and supratemporal gyri, the regions around the arcuate and principal sulci, the inferior colliculus, inferior pulvinar, or reticular nucleus of the thalamus is capable of eliciting a lever-pressing $C R$ for shock-avoidance. With sufficient training a single $1.0 \mathrm{msec}$. pulse of $1.0 \mathrm{mAmp}$., applied to the occipital lobe, or $0.1 \mathrm{mAmp}$. to the region of periaqueductal gray and the third nerve nuclei, elicit a CR in the monkey. There is no reason the same should not be true for man, and it will be of great interest to see what relation such evoked, "learned" neural activity bears to conscious processes.

From these diverse facts, as discussed elsewhere in more detail, ${ }^{5,6,9,11}$ it is possible to imagine that the basic factor in the formation of conditioned reflexes is a change in the threshold of a subcortical neural hierarchy controlling the complex conditioned movement. The existence of such controlling hierarchies at subcortical levels is well documented. These hierarchies range from the scratch reflex and stepping movements at spinal levels to postural, righting, and attitudinal reflexes organized in the mesencephalon; or the chewing, licking, and swallowing reflexes of the mesencephalon and medulla compounded in the feeding patterns elicited by hypothalamic stimulation. It is probable that some of the hierarchies controlling conditioned movements have a cortical link in their organization, or that cortical activity contributes importantly to the threshold shifts in the controlling neurons. An example of the latter action may perhaps be seen in the experiments of Rusinov ${ }^{22}$ and of Morrell, reported in this volume, whereby the polarization of the motor cortex so alters the threshold of a particular movement complex that it can be elicited by photic or auditory stimuli. Possibly in an analogous fashion the application of a CS anyplace in the nervous system contributes sufficient additional excitation to the neurons controlling the conditioned movement (whose threshold has somehow been lowered by training and the experimental situation) to trigger the CR.

The evidence cited above points to the importance of subcortical elements in $\mathrm{CR}$ elicitation. Much or most of the organization of movements occurring to 
cortical stimulation must arise in subcortical hierarchies. Production of an integrated movement by cortical stimulation would be unlikely if the organization were effected at the cortical level. The stimulating current should disrupt any normal processes of integration inherent in the stimulated cortex, yet integrated movements can be elicited. A movement of the hand up, opening the mouth, and retraction of the tongue occurs often in infants at the "oral stage." A highly similar movement pattern of mouth and arm can be elicited by stimulation of the monkey precentral gyrus ${ }^{6,11}$ or rhinal fissure. ${ }^{8}$ This shows that a naturally occurring primate movement pattern can be elicited by presumably disruptive cortical stimulation and, as in the case of other movements "inherent" in cats ${ }^{11}$ and of similarly conditioned movements in cats, dogs, and mon. keys, this pattern can be elicited by stimulation at more than one location. One cannot easily believe the movement to be organized at each location of the stimulating electrodes.

Furthermore, as shown by Loucks ${ }^{15}$ and confirmed in our experiments on monkeys, the movement elicited by cortical stimulation can be altered by training: indeed, as is obvious from the above, through conditioning procedures stimulation in any selected cortical area can elicit the same (conditioned) movement. This holds true even for the monkey precentral gyrus, in which stimulation that inherently yields an arm flexion can, after training, produce an extension. (Actually, however, the situation is not this simple, since stimulation of the precentral gyrus will still produce arm flexion after training if the current is increased.) The extensor movement is elicited with currents too low to elicit any inherent movement, and one is tempted to say in subjective terms that the perceptual or sensory threshold for the "motor" cortex is lower than that for eliciting movement. Nevertheless the motor system inherently activated from this area is also being affected by this low, tetanizing current, since it is thrown into immediate action by a loud, unusual noise or, especially, by a painful stimulus. The effect of the latter completely overwhelms the conditioned extensor movement and the cortical stimulus, previously subliminal for the inherent movement, then sums with the painful input from the tail to produce arm flexion of convulsive intensity. Again it seems unlikely that the neural site of this convergence of excitation applied to tail and cortex is beneath the cortical electrodes.

The painful stimulus brings into play complex emotional factors that might underlie this augmentation. Our Soviet colleagues ${ }^{21}$ caution us that in the use of these concepts of emotion and motivation we take care to avoid the implication that it is the concomitant psychic activity that influences the neural outcome. To the materialist, however, this is a completely artificial problem, since the psychic activity is but a manifestation of neurons and hence, obviously, cannot interact with them. In speaking of neural behavior in which the complexity of action lies beyond our present capacity to analyze it, I see no prejudice whatever to the materialistic argument to speak of these behaviors in terms of their probable psychic manifestations, of the totalized and integrated neural pattern. Naturally this adds nothing to our understanding of the neural basis of this activity, but it is nevertheless useful in that it enables us to describe and think about this activity in common sense terms and to keep before us a problem of major importance in behavioral neurophysiology. 
Certain forms of neural activity, inherently rewarding or punishing, pleasant or unpleasant, positively or negatively reinforcing or motivating, or biologically significant, are able to organize the entire motor apparatus into activities of approach or avoidance and to exert great influence upon the systems operative in memory and learning. MacLean ${ }^{16}$ has emphasized that the limbic system is the seat of this motivational activity, and Olds ${ }^{19}$ and Lilly, ${ }^{14}$ among others, have further confirmed this by using the animal's reaction of starting or stopping trains of electrical stimuli in these regions as an index of inherent motivational connections. There is some danger, of course, that the self-stimulation achieved in such procedures is an experimental artifact resulting from the confusional effects of electrical stimulation of the limbic system. In most instances a much higher intensity of current is required for self-stimulation than for elicitation of defensive $\mathrm{CRs}^{5,17}$ or of arousal (Olds, cited by Doty ${ }^{4}$ ) from the same point, and electrical abnormalitites are often induced by this high intensity self-stimulation..$^{5,20}$

Sometimes, however, very low intensities are able to maintain self-stimulation (Olds and Lilly, cited by Doty ${ }^{4}$ ), and there is no a priori reason why one should not be able, with an electrical stimulus, to enter positively motivating systems. Of 21 cats tested in our laboratory ${ }^{5,17}$ (unpublished data) with electrodes in 70 points, primarily within the limbic system, aversive effects seemed predominant. Self-stimulation was obtained from only 13 locations, whereas stimulation at another 37 positions was avoided. The lowest threshold found for self-stimulation was $0.5 \mathrm{mAmp}$. for a $0.5 \mathrm{sec}$. train of $0.2 \mathrm{msec}$. pulses at 300 pulses/second in cats 370 and 438 . In cat 438 the electrodes were aimed at the anterior commissure and in cat 370 at the anterior ventral nucleus of the thalamus. Self-stimulation rates in both animals were higher with 1.0 to $1.5 \mathrm{mAmp}$. In cat 438 the threshold subsequently found for eliciting a flexion CR was 0.4 mAmp. In each case the self-stimulation was abruptly slowed or halted when a moderately aversive stimulus of similar timing followed or preceded the 1.0 to $1.5 \mathrm{mAmp}$. rewarding stimulus by a few tenths of a second. Thus the selfstimulation seemed to produce neither "confusion" nor automatic, driven behavior.

A threshold of $0.5 \mathrm{mAmp}$. for self-stimulation at rates of $1000 \mathrm{presses} /$ hour, comparable to those for cats 370 and 438 , was also obtained in one cynomolgous monkey, not from the limbic system but from electrodes histologically localized in the right inferior pulvinar and brachium of the superior colliculus! The animal could be awakened by a stimulus train of as little as $0.2 \mathrm{mAmp}$. Each stimulus burst at the 1.0 to $1.5 \mathrm{mAmp}$. level usually employed for self-stimulation made the eyes flick about $30^{\circ}$ horizontally to the left. Light flashes evoked potentials in this region and, if the animal was in the dark, single electrical pulses elicited potentials in the ipsilateral occipital lobe; yet the animal would not self-stimulate while in the dark. If bursts of stimuli were administered in rapid succession while the experimenter was with the animal, its eyes shifted each time under the influence of the stimulus but were immediately brought to bear again as though its attention was undisturbed. Thus, in this case too, it seems safe to surmise that the self-stimulation somehow produces satisfaction rather than confusion, but the nature of satisfaction from stimulation within 
the visual system is presently as indescribable in neural terms as is an esthetic experience.

In addition to its usefulness in studying motivational systems, the self-stimulation procedure is equally important in showing that stimulation of many regions of the nervous system, particularly the cerebral cortex, is without motivational effect. Thus Giurgea and $\mathrm{I}^{11}$ were able to show during our brief opportunity to work together that the cortical stimuli he used as both CS and US to establish behavioral CRs ${ }^{12,13}$ were without effect on the animal's behavior in a self-stimulation situation. In other words, by his technique of corticalcortical stimulus pairing, conditioned reflexes of the usual behavioral type can be formed with stimuli that lack any demonstrable motivational component. The conditioning mechanism per se may thus operate independently of the motivational factors that are unavoidable in most behavioral conditioning procedures. The motivational component, however, probably plays a very important role when it is present.

Direct comparison of the motivated versus nonmotivated reflexes has thus far been made in one monkey. In this instance a $\mathrm{CR}$ of arm flexion was established by pairing stimulation of the visual cortex as CS with stimulation of the motor cortex as US."1 The animal in the self-stimulation procedure was entirely indifferent to these stimuli. The threshold for CR elicitation from visual cortex stimulation was $0.55 \mathrm{mAmp}$. in this nonmotivated situation. The same visual cortex CS was then paired with an electric shock to the tail, which could be avoided by a lever-press during the CS. After training with this highly motivating US, only $0.2 \mathrm{mAmp}$. was required for stimulation of the visual cortex to elicit a lever-pressing CR. The motivational system can be inferred to be responsible for this altered threshold for effective cortical stimulation and, in addition, is probably also operative in shifting the elicited movement from flexion to extension in the absence of any specific movement pattern imposed by the US.

In summary, subcortical systems predominate in effecting the attentive and motivational components so important in most conditioning. A major portion of the motor system representing the conditioned reflex itself may be organized subcortically and be the site of the principle change in excitability resulting from the conditioning process. The cortex in such a scheme would contribute to the motor organization and the background shifts of excitability in the conditioned system, but would serve principally as the analyzer of the sensory inputs. Beyond stating that both cortical and subcortical elements when present must always be involved in conditioned reflex formation, the available data and our understanding of their significance are still too vague to support much specific speculation on what is occurring in this complex process.

\section{References}

1. BECK, E. C., R. W. Doty \& K. A. Koor. 1958. Electrocortical reactions associated with conditioned flexion reflexes. EEG Clin. Neurophysiol. 10: 279-289.

2. BECK, E. C., M. RudNIK \& P. B. PorTER. Cortical and subcortical electrophysiological responses during the latent interval of a delayed response. In preparation.

3. Delgado, J. M. R. 1959. Prolonged stimulation of brain in awake monkeys. J. Neurophysiol. 22: 458-475. 


\section{Doty: On the Role of Subcortical Structures}

4. Doty, R. W. 1959. Brain stimulation and conditional reflexes. In The Central Nervous System and Behavior. Trans. 1st Conference. M. A. B. Brazier, Ed. Josiah Macy, Jr. Foundation. New York, N. Y.

5. DoтY, R. W. Conditioned reflexes formed and evoked by brain stimulation. In Electrical Stimulation of the Brain. D. E. Sheer, Ed. Univ. Texas Press. Houston, Texas. In press.

6. Doty, R. W. 1960. Neural hierarchies and behavior. In The Central Nervous System and Behavior. Trans. 3rd Conference. : 414-421. M. A. B. Brazier, Ed. Josiah Macy, Jr. Foundation. New York, N. Y.

7. Doty, R. W., L. T. Rutledge \& R. M. LARsen. 1956. Conditioned reflexes established to electrical stimulation of cat cerebral cortex. J. Neurophysiol. 19: 401-415.

8. Doty, R. W., E. C. BECK, \& K. A. KooI. 1959. Effect of brain stem lesions on conditioned responses of cats. Exptl. Neurol. 1: 360-385.

9. Doty, R. W. \& L. T. Rutledge. 1959. "Generalization" between cortically and peripherally applied stimuli eliciting conditioned reflexes. J. Neurophysiol. 22: 428435 .

10. Doty, R. W. \& L. T. Rutledge. 1959. Conditioned reflexes elicited by stimulation of partially isolated cerebral cortex. Federation Proc. 18: 37.

11. DotY, R. W. \& C. GiurgeA. 1961. Conditioned reflexes established by coupling electrical excitation of two cortical areas. In Brain Mechanisms and Learning. J. Delafresnaye, Ed. Blackwell Scientific Publ. London, England. In press.

12. Giurgea, C. 1953. Elaborarea Reflexului Conditionat prin Excitarea Directa a Scoartei Cerebrale. 154 pp. Editura Academia Rep. Pop. Romane. Bucharest, Rumania.

13. Giurgea, C. \& N. Raiciulescu. 1959. Etude électroencéphalographique du réflexe conditionnel à l'éxcitation électrique corticale directe. In 1st Internat. Congr. Neurol. Sci. 3: 156-176. L. van Bogaert and J. Radermecker, Eds. Pergamon. London, England.

14. LILLY, J. C. 1958. Learning motivated by subcortical stimulation: the start and stop patterns of behavior. In Reticular Formation of the Brain. : 705-721. H. H. Jasper, L. D. Proctor, R. S. Knighton, W. C. Noshay and R. T. Costello, Eds. Little, Brown. Boston, Mass.

15. Lovcks, R. B. 1935. The experimental delimitation of neural structures essential for learning: the attempt to condition striped muscle response with faradization of the sigmoid gyri. J. Psychol. 1: 5-44.

16. MACLEAN, P. D. 1959. The limbic system with respect to two basic life principles. In The Central Nervous System and Behavior. Trans. 2nd Conference. : 31-118. M. A. B. Brazier, Ed. Josiah Macy, Jr. Foundation. New York, N. Y.

17. Nielson, H. C., R. W. Doty \& L. T. Rutledge. 1958. Motivational and perceptual aspects of subcortical stimulation in cats. Am. J. Physiol. 194: 427-432.

18. Nielson, H. C., J. M. KNIGH \& \& P. B. Porter. Subcortical conditioning, generalization, and transfer. In preparation.

19. Olps, J. 1958. Adaptive functions of paleocortical and related structures. In $\mathrm{Bi}$ ological and Biochemical Bases of Behavior. : 237-262. H. F. Harlow and C. N. Woolsey, Eds. Univ. Wis. Press. Madison, Wis.

20. Porter, R. W. D. G. Conrad \& J. V. Brady. 1959. Some neural and behavioral correlates of electrical self-stimulation of the limbic system. J. Exptl. Analysis of Behavior. 2 : 43-55.

21. Sarkisov, S. A., V. S. Rusinov \& M. Y. Rabinoivitch. 1960 . Book review of Central Nervous System and Behavior. EEG Clin. Neurophysiol. 12: 172-174.

22. Rusinov, V.S. 1953. An electrophysiological analysis of the connecting function in the cerebral cortex in the presence of a dominant region area. Abstr. Communications XIX Internat. Physiol. Congr. Montreal. : 719. 